\title{
Landmark Guided Forwarding
}

\author{
Menghow Lim ${ }^{\S *}$ Adam Greenhalgh ${ }^{\ddagger \dagger}$ Julian Chesterfield ${ }^{\S}$ Jon Crowcroft ${ }^{\S}$ \\ ${ }^{\S}$ Computer Laboratory, University of Cambridge, UK \\ \{meng.how - lim, julian.chesterfield,jon.crowcroft $\} @$ cl.cam.ac.uk \\ ${ }^{\ddagger}$ Dept. of Computer Science, University College London, UK \\ a.greenhalgh@cs.ucl.ac.uk
}

\begin{abstract}
In this paper we focus on the problems of maintaining Ad Hoc network connectivity in the presence of node mobility whilst providing globally efficient and robust routing. The common approach among existing wireless Ad Hoc routing solutions is to establish a global optimal path between a source and a destination. We argue that establishing a globally optimal path is both unreliable and unsustainable as the network diameter, traffic volume and number of nodes all increase in the presence of moderate node mobility. To address this we propose Landmark Guided Forwarding $(L G F)$, a protocol that provides a hybrid solution of topological and geographical routing algorithms. We demonstrate that LGF is adaptive to unstable connectivity and scalable to large networks. Our results indicate therefore that Landmark Guided Forwarding converges much faster, scales better and adapts well within a dynamic wireless Ad Hoc environment in comparison to existing solutions.
\end{abstract}

\section{Introduction}

Ad Hoc networking is a topic of widespread interest amongst the network and systems research communities of late due to the novel challenges associated with providing truly distributed and decentralised communication architectures. Building Ad Hoc networks over wireless links introduces even more complexity due to the irregular and unpredictable nature of the wireless medium.

The primary objective of wireless Ad Hoc networks is to enable a set of highly cooperative wireless nodes to establish communication quickly without any fixed infrastructure. In addition to sending and receiving packets, each

\footnotetext{
* Menghow Lim's PhD work is funded by the Cambridge-MIT Institute

†Adam Greenhalgh's post doctorial research is funded by the Cambridge-MIT Institute
}

node also acts as a relay for packets travelling across the network from a source which may not be able to directly access the destination node, for example, as a result of signal power limitations, or due to the well known 'hidden node' problem.

Unlike mobile hosts in an infrastructure based mobile network, such as an office or home setting with a dedicated wireless base station, nodes in a Mobile Ad Hoc Network (MANET) must collectively manage communication in a cooperative fashion. MANETs rely on the common sharing of resources to achieve a collective goal.

There are a variety of issues and solutions surrounding the development of social and economic models to provide incentives for cooperative network architecture formation, such as is required in the Ad Hoc scenario, which we do not address in this paper. Our work concerns the mechanics for Ad Hoc routing, and as such builds upon a substantial body of research. However, our work differs from existing approaches in a number of respects.

Whilst many routing protocols utilise either topologically driven route optimisation, or geographically driven route optimisation, we maintain that a more efficient approach is to leverage benefits from each, creating a hybrid approach towards routing, optimised around various local and global parameters. In this paper we present Landmark Guided Forwarding (LGF), a novel approach to Ad Hoc network routing that achieves the following:

- Lower average routing state maintenance across the node set.

- Reduced the spread of routing updates.

- Reduced stale routing entries.

- Adaptive to dynamic Ad Hoc mobility.

Unlike topological routing protocols such as DSDV, DSR and AODV [4, 5, 14], Landmark Guided Forwarding requires that every node only maintains a small amount 
of topological and position information about neighbours within a localised area. Routing is achieved by using locally optimised algorithms, requiring lower network overhead. If the packet destination resides within the local area, it is routed using the shortest path algorithm. Otherwise, when the destination resides outside the local scope, it is routed towards a geographically determined optimal Landmark node. Unlike position based forwarding schemes such as GPSR and Face routing [1][3], LGF does not rely upon the establishment of planar graph, but leverages on the local hybrid routing information available, thereby increasing the resilience to inconsistent device position information and lowering the overall system vulnerability to position errors [8].

In the remainder of this paper, we state the assumptions we have made while developing LGF. We follow on to describe LGF in detail and examining similar related work in the field before describing how we simulated LGF in different scenarios. Finally we summarise the results and conclude before suggesting some possible future extensions to this work.

\section{Assumptions}

We make a few assumptions commonly used by position based forwarding protocols. We assume that every node knows its own geographic position. This is not an unreasonable assumption since it is feasible to gather position information from GPS or another positioning system. Since LGF does not require high precision, short range distance measurements from Bluetooth devices or via IEEE 802.11 based ranging systems such as the Intel Place Lab system [9] are suitable alternatives to a GPS based system. We also assume a distributed location service like the Grid Location Service[11] is available for a source node to retrieve the geographic position of a destination node.

\section{Protocol description}

Ad Hoc networks rely on nodes in the network to relay packets between a source and a destination on behalf of their peers. As a packet flows between the source and destination, LGF calculates the locally optimal path to the destination and applies the shortest path to the destination if it is within the local area. In cases where the destination is not within the local area, LGF employs locally optimal routing towards the node that is geographically closest to the destination. The protocol iterates progressively. Once the packet is forwarded, it will reveal a new set of neighbours and a local optimal route towards the destination. Using this technique not only effectively sidesteps the scalability constraints associated with global optimal routing as used by existing MANET protocols, but also allows routing to be more adaptive to the ever changing MANET topology. The approach taken by LGF only requires advertisement of topological and geographical information to a node's neighbours that are within a few hops. Thus it localises state dissemination and reduces the overall load on the network. This also allows localised state to converge much faster by adaptive updates that regulates the neighbourhood state update frequency based on the surrounding network connectivity. In essence, these properties allow LGF MANETs to be extended to a larger environment and be more adaptive to dynamic Ad Hoc mobility than other MANET protocols.

In this section, we present various algorithms that form Landmark Guided Forwarding. The protocol consists of various components, namely: restrictive hybrid route advertisement, adaptive route advertisement, link failure recovery, next hop selection, path exploration, dead-end detection and loop avoidance. We describe each of these in turn in the later sections.

\subsection{Restrictive hybrid route advertisement}

In order to retain a balance between timeliness of routing decisions and the overhead of route advertisements, we propose a pro-active routing scheme based on a localised hybrid routing table. Using this approach, information about a node's geographical position and local topology is disseminated to a limited topological area. We define each node's neighbours to be within a topological area defined by the perimeter $P$ in number of hops. For each neighbour node $j$ within $P$, node $i$ maintains its position, $x_{j}, y_{j}, z_{j}$, and additional information as a routing entry $R E_{i j}$, in the routing table $R T_{i}$. A routing entry $R E_{i j}$ is given below:

$R E_{i j}=\left\{j\right.$, NextHop $_{i j}$, HopCount ${ }_{i j},\left\{x_{j}, y_{j}, z_{j}\right\},\left\{\dot{x}_{j}\right.$, $\left.\dot{y}_{j}, \dot{z}_{j}\right\}$, Seq.Num$\left.\cdot_{\cdot i j}\right\}$

Where $j$ is the destination and is a globally unique node identifier of all nodes within $P$, and the NextHop is the identifier of adjacent node that a packet should be forwarded to in order to reach the destination which is HopCount hops away. A sequence number Seq.Num $\cdot_{i j}$ is associated with each entry to ensure timeliness. The position and velocity of the destination $j$, are $\left\{x_{j}, y_{j}, z_{j}\right\},\left\{\dot{x}_{j}, \dot{y}_{j}, \dot{z}_{j}\right\}$ respectively. These attributes are used by the forwarding algorithm to resolve a local optimal path when destination address $d s t_{p}$ of a packet $p$ is not in $R T_{i}, \forall j, d s t_{p} \neq j$.

In order to explain the restricted hybrid routing advertisement process with node mobility, we use an example. Figure 1 shows a small Ad hoc network scenario where node 3 moves from its central position to a new position in the top right of the network, all other nodes remain stationary. We demonstrate the scheme by comparing the routing tables and the topological view of the network from node 5 's point of view. 


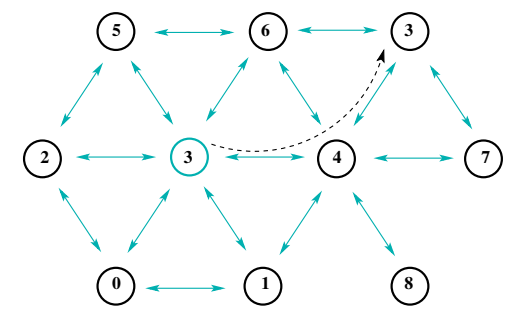

Figure 1. Mobility scenario in an Ad Hoc network

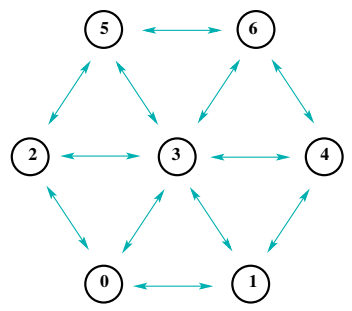

Figure 2. Node 5's topological view of the network before node 3 moves.

In this example, the restrictive hybrid advertisement does not propagate more than 2 hops from source and therefore nodes 7 and 8 are not included in node 5 's routing table, table 1, and its topological view of the network, as illustrated in figure 2 before node 3 movement.

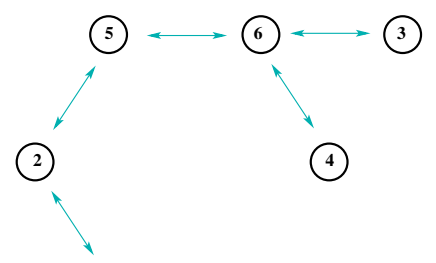

(0)

Figure 3. Topological view of node 5 after node 3 move away

If we re-examine node 5's routing table, table 2 , and its topological view of the network, figure 3 , after the movement of node 3 . Node 1 is now no longer routable using local optimal routing, nodes 3 and 4 are now only routable via node 6 and node 0 is only routable via node 2 .

In this scenario, node 3's movement causes the routing algorithm to make the following adjustments to the routing table of node 5 , i.e. table 1 is transformed to table 2 .

- Remove entries for destinations which have a hop count greater than 2 .
Table 1. Node 5's routing table before node 3 moves.

\begin{tabular}{llllll}
\hline Dst & Next Hop & Metric & $x$ & $y$ & $z$ \\
\hline 0 & 2 & 2 & 300.00 & 2.00 & 0.00 \\
1 & 3 & 2 & 450.00 & 2.00 & 0.00 \\
2 & 2 & 1 & 225.00 & 132.00 & 0.00 \\
3 & 3 & 1 & 375.00 & 132.00 & 0.00 \\
4 & 3 & 2 & 525.00 & 132.00 & 0.00 \\
5 & 5 & 0 & 300.00 & 262.00 & 0.00 \\
6 & 6 & 1 & 450.00 & 262.00 & 0.00 \\
\hline
\end{tabular}

Table 2. Routing table of node 5 after node 3 move Away

\begin{tabular}{llllll}
\hline Dst & Next Hop & Metric & $x$ & $y$ & $z$ \\
\hline 0 & 2 & 2 & 300.00 & 2.00 & 0.00 \\
2 & 2 & 1 & 225.00 & 132.00 & 0.00 \\
3 & 6 & 2 & 600.00 & 262.00 & 0.00 \\
4 & 6 & 2 & 525.00 & 132.00 & 0.00 \\
5 & 5 & 0 & 300.00 & 262.00 & 0.00 \\
6 & 6 & 1 & 450.00 & 262.00 & 0.00 \\
\hline
\end{tabular}

- Update of location information.

- Update of next hop and metric information.

Specifically it can be observed that the entry for node 1 has been removed from the routing table of node 5 in table 2. The position of node 3 has been updated. The next hop and metric of nodes 3 and 4 have also been updated accordingly.

\subsection{Next hop selection algorithm}

Our approach is to take advantage of the geographical position of those nodes that are within each node's topological scope as a basis for the forwarding algorithm. Each node $i$ maintains the topological distance HopCount ${ }_{i j}$ and position $x_{j}, y_{j}, z_{j}$ for every other node $j$ that is within its scope. The next hop is selected using the shortest path algorithm to each packet's destination $d$, where $d$ matches one of the neighbours $j$. Otherwise, the next hop is determined by Landmark Guided Forwarding that selects the next hop by applying shortest path to a landmark node $V$. Where node $V$ is geographically closer to the destination node $D$ and topologically further away from node $i$. The term Landmark has been widely used to describe a physical point of reference for an Internet coordinate system [17]. In this paper, the Landmark is a temporary reference node amongst the collection of $j$, that acts as a virtual destination to assist in the 
routing of a packet towards its final destination. The exploration algorithm is progressive, as soon as the packet moves to the next hop, a new Landmark node amongst the new set of neighbours is determined and the packet progresses in the same manner until it arrives at a node with a topological path to the destination. However, in the case where no valid Landmark node is available for forward advancement, the path exploration algorithm rolls back and seeks an alternate path from the previous hop.

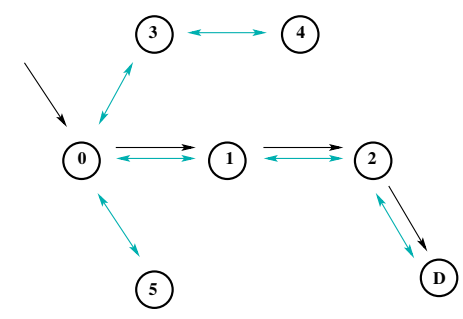

Figure 4. Next hop selection

Figure 4 shows a subgraph that demonstrates our forwarding algorithm where the topological scope is limited to 2 hops. If we consider the packet arrives at node 0 destined for node 4 , it can be forwarded to the destination via either node 1 , node 3 or node 5, by applying the shortest path algorithm to the destination, the next hop is found to be node 3 . In the case where a packet's destination is not within the coverage of the topological scope, the next hop is chosen by the shortest path algorithm to a landmark node. For this example in figure 4 , the next hop is node 1 since node 2 is found to be closer to the destination than node 4 .

\subsection{Path exploration}

In general, geodesic proximity to the destination does not assure a shorter topological path to the destination. Simply forwarding a packet towards its destination position without maintaining any forwarding path history does not provide any facility for preventing the packet being trapped by a localised loop or dropped due to a routing dead-end and subsequently backtracking. The approach adopted in our algorithm is to include a source path in the packet header and to also maintain soft forwarding state amongst all nodes traversed by a packet. By maintaining a source path in the packet header it provides a trail of forwarding nodes such that in the event a dead-end is encountered, the packet can be back-tracked until it reaches a node with an alternative path to the destination. In addition, this also enables the algorithm to preserve its loop free property by not selecting a virtual landmark or next hop that is the source path. The purpose of maintaining soft-state within the network is to isolate and explore the network systematically. A node temporarily marks a link with the tuple
\{Packet_Sequence_Number, Next_Hop,SSoft_State_Expiry\}, once it has forwarded a packet along that link. This enables the exploration algorithm to search all available paths and guarantee packet delivery where a path is available between a source and destination.

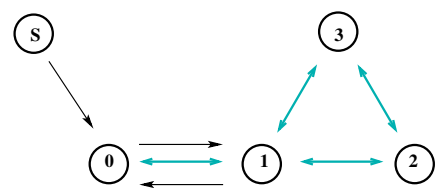

Figure 5. Dead end detection and roll back

Figure 5 shows a subgraph that demonstrates how a dead end can be detected while a packet systematically explores a path to the destination. In this scenario, a packet from node $S$ arrived at node 0 . Assume the packet's destination is not reachable by any node in the figure. In addition, the destination is geographically closer to node 2 than node 3 , the topological scope being 2 hops. We denote $S P$ as a sequence of nodes in the source path. At node 0 , where $S P=(S)$, we determine the next Landmark node, according to our next hop selection algorithm, as node 2 . The next hop node chosen to forward the packet towards node 2 is node 1 based on the shortest path algorithm.

When the packet arrives at node $1, S P=(S, 0)$, it becomes apparent that the only node that is 2 hops away from node 1 is $S$. However, since $S$ is found in the source path $S P$, the algorithm considers $S$ to be an invalid landmark. With no available landmark and the packet's destination not within the topological range, the path exploration detects that the packet is moving towards a dead-end and retracts the packet back to node 0 . In this example, node 0 established soft-state when the packet was forwarded from node 0 to node 1 and likewise node $S$ had established soft-state when the packet was forwarded from node $S$ to node 0 . Retracting back to node 0 , the packet's source path $S P$ is shortened to $(S)$. At this point, the path exploration is aware that the link between node 0 and node 1 has already been visited. Since there is no forwarding path available, the packet is pulled back to node $S$. With no other link available at node $S$, the path exploration has exhausted all searches and drops the packet.

Figure 6 shows a subgraph that demonstrates how a loop is avoided while a packet explores a path to its destination. In this scenario the topological scope is 2 hops and the source node is $S$. The destination $D$ is not directly connected to any node in the subgraph. Based on our next hop selection algorithm, the packet at node $S$ identifies node 2 as its Landmark node. Following the shortest path algorithm to node 2 , the packet is directed towards node 1 . Subsequently, the packet is forwarded to node 2 with Landmark node 4 . 


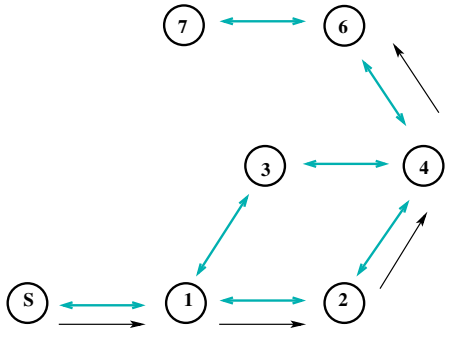

Figure 6. Loop avoidance

The same process is repeated when the packet moves from node 2 to node 4 with node 3 as its respective Landmark node. When the packet arrived at node 4 , it found $S P=(S, 1,2)$ with both node 1 and 7 at its' topological range, i.e. within 2 hops of node 4 . With node 1 in its' source path, the algorithm provides only one option of forwarding towards node 6 with node 7 as the Landmark node. This effectively avoids the creation of a loop between $1 \rightarrow 2 \rightarrow 4 \rightarrow 3$.

\subsection{Link failure recovery}

When a node moves out of range of it's neighbours, established links are likely to break. Typically a broken link may be detected either by the link layer protocol timing out a connection, or it may be inferred at a higher level through the loss of a periodic broadcast signal which is expected within a predefined time. In our protocol, a node represents a broken link with $\infty$.

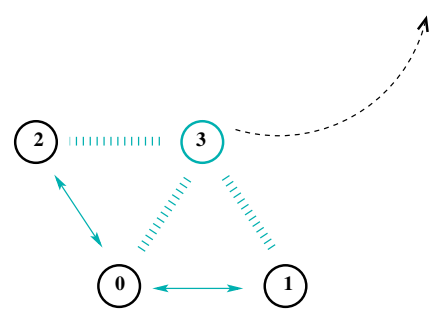

Figure 7. State propagation and maintenance

Figure 7 illustrates a mobility scenario in which node 3 moves out of range of nodes 0,1 and 2. Node 1 is initially a neighbour of node 3 , and records a route to node 3 with a metric of 1 as shown in table 3 . As node 3 moves out of range, the node detects the loss of a link, and updates it's table accordingly. Table 4 illustrates the change in routing metrics; the routes to both node 3 and node 2 which originally travelled via 3 are set to $\infty$. Node 1 subsequently broadcasts these routing entries to all it's single hop neighbours. Once the routing state has been synchronised in this manner, the node performs a periodic state mainte- nance process, removing or replacing the entries of $\infty$ metrics with cheaper routes as illustrated in table 5 .

\section{Table 3. Routing table of node 1 before link broken}

\begin{tabular}{llllll}
\hline Dst & Next Hop & Metric & $x$ & $y$ & $z$ \\
\hline 0 & 0 & 1 & 300.00 & 2.00 & 0.00 \\
1 & 1 & 0 & 450.00 & 2.00 & 0.00 \\
2 & 3 & 2 & 225.00 & 132.00 & 0.00 \\
3 & 3 & 1 & 375.00 & 132.00 & 0.00 \\
\hline
\end{tabular}

Table 4. Routing table of node 1 after link broken

\begin{tabular}{llllll}
\hline Dst & Next Hop & Metric & $x$ & $y$ & $z$ \\
\hline 0 & 0 & 1 & 300.00 & 2.00 & 0.00 \\
1 & 1 & 0 & 450.00 & 2.00 & 0.00 \\
2 & 3 & $\infty$ & 225.00 & 132.00 & 0.00 \\
3 & 3 & $\infty$ & 375.00 & 132.00 & 0.00 \\
\hline
\end{tabular}

\section{Table 5. Routing table of node 1 after state maintenance}

\begin{tabular}{llllll}
\hline Dst & Next Hop & Metric & $x$ & $y$ & $z$ \\
\hline 0 & 0 & 1 & 300.00 & 2.00 & 0.00 \\
1 & 1 & 0 & 450.00 & 2.00 & 0.00 \\
2 & 0 & 2 & 225.00 & 132.00 & 0.00 \\
\hline
\end{tabular}

\subsection{Adaptive route advertisement}

One key feature of LGF is its ability to regulate the restrictive hybrid update frequency according to its interconnectivity with other adjacent nodes within its radio range. The essence of this feature is to associate update frequency with the furthest adjacent node. The greater the distance between node and its furthest adjacent node, the more frequently the node must send out its routing updates. This increase in the rate of state propagation enables the network to converge much faster when adapting to changes in the surrounding network connectivity.

\section{Related work}

Many wireless Ad Hoc wireless routing protocols have been proposed in recent years. An early survey paper [13] categorised these protocols as table driven or source driven. In general, table driven protocols pro-actively gather topological routing information while source driven protocols 
reactively discover a route or routes to the destination as requested by the source. Pro-active routing protocols such as DSDV, Destination Sequenced Distance Vector [4], proactively exchange routing information between neighbouring nodes. The associated routing state and the network traffic overheads is $O(n)$, where $\mathrm{n}$ is the number of nodes in the network, which does not scale well in large networks. Reactive routing protocols such as AODV [14], Ad Hoc on Demand Distance Vector, use flooding techniques to discover new routes and repair existing routes. As the amount of traffic in the network increases or the diameter of the network increases, the cost of flooding increases. With reactive routing protocols, the routing performance degrades under moderate mobility conditions [10][16].

An alternative approach to Ad Hoc routing is to take advantage of the physical location of nodes in the network and to do position based forwarding. An assumption made by protocols that take this approach is that every node knows its own geographical position. By limiting the exchange of positional information to be only between adjacent nodes, the state and network overheads are reduced to $O(u)$, where $\mathrm{u}$ is the number of adjacent nodes. GPSR, Greedy Perimeter Stateless Routing [1], is a position based routing protocol that in general uses the geographically closest node to the destination as the next hop for the packet to be forwarded. However, in a local maximum scenario, this technique can prevent greedy forwarding from advancing towards the destination. To address a situation like this, GPSR uses a perimeter forwarding scheme that uses the well known right hand rule on its planarised graphs. Although GPSR scales well and is able to adapts to random topologies, it is vulnerable to position errors. A recent research article suggests that position inconsistencies in position based forwarding protocols could cause false greedy forwarding and misconstruction of the planar graph [8]. The results show various position inconsistencies do have significant negative effects on the performance of position based routing protocols. In LGF, position information is used together with a heuristic technique to explore the network systematically, our report [12] shows this approach is able to sustain significant position inconsistency without degrading routing performance.

LGF is similar in some of its features to existing routing protocols, such as ZRP [7] and Terminode [2]. In common with these two protocols, LGF uses a hierarchical framework that employs two different routing schemes. Each node pro-actively maintains connectivity with other nodes within its neighbourhood. A packet is routed using the shortest path algorithm when the destination is within this neighbourhood. In contrast, a packet destined for outside the local neighbourhood is routed using a more scalable routing protocol. ZRP uses reactive routing to determine the optimal path to destination whereas Terminode uses position based forwarding to forward the packet towards the direction of destination. In contrast to ZRP which uses a flooding technique to discover the destination, LGF progressively uses hybrid routing to explore the network systematically when delivering packets to the destination. In the case of the Terminode routing protocol, it uses greedy forwarding to forward packets, but it requires some static nodes to establish stable paths when greedy forwarding is not applicable. Conversely, LGF requires no static node, and its exploration algorithm is able to avoid looping and dead-ends even in the presence of high rates of mobility.

\section{Simulation scenario}

The simulations have been carried out using the NS2 simulator [6], with each simulation lasting for 900 seconds. Each node uses the IEEE 802.11 MAC and the physical model models the radius of the radio range as being 250 meters. The simulation uses the random way point model to model node mobility. In all simulation scenarios, each node selects a random destination and moves at a speed uniformly distributed between 0 and maximum velocity. Upon reaching the destination, the node pauses for a configured period before it selects the next random destination and moves on. The traffic model uses constant bit rate UDP traffic flows, with 512 byte payloads. The start time for the different flows is uniformly distributed between 0 and 180 seconds with each of the 30 traffic sources sending at the rate of 2 packets per second. In common with other protocol evaluations, [1][4][14][5], we run several mobility patterns with different pause times at a constant maximum velocity. We use 5 different sets of mobility patterns generated with different pause times of 0,30,60,120,600 and 900 seconds where the maximum velocity is $15 \mathrm{~m} / \mathrm{s}$. This simulation is run in a geographic area of $1500 \times 300 \mathrm{~m}^{2}$ with 50 nodes randomly placed. We also run simulations using different maximum velocities $(1,2.5,5,7.5,10,12,5,15 \mathrm{~m} / \mathrm{s})$ where the nodes move continually, with a pause time of 0 seconds. This second set of simulations is run in an area of 1500x $500 \mathrm{~m}^{2}$ with 100 nodes randomly placed in the area. We compare LGF with DSDV, AODV and GPSR using the different simulation scenarios we have just described and we compare the adaptability, performance and overheads of LGF with other MANET routing protocols. Each of the different MANET routing protocols has some settings specific to it, we detail these in table 6,7,8 and 9.

\section{Results}

The results are divided into three subsections: performance with varying pause time, performance with varying velocity and path length. 
Table 6. GPSR specific parameters

\begin{tabular}{ll}
\hline Parameter & Value \\
\hline Beaconing interval & $3 \mathrm{~s}$ \\
Random variation of beaconing interval & $0.5 \%$ \\
Beacon expiration interval & $13.5 \mathrm{~s}$ \\
Promiscuous mode & enable \\
Removal of neighbor from neighbor list & enable \\
when link broken & \\
Perimeter mode & enable \\
\hline
\end{tabular}

Table 7. DSDV specific parameters

\begin{tabular}{ll}
\hline Parameter & Value \\
\hline Initial weight settling time & $6 \mathrm{~s}$ \\
Periodic update interval & $15 \mathrm{~s}$ \\
$\begin{array}{l}\text { Number of missed periodic updates } \\
\text { before declaring link broken }\end{array}$ & 3 \\
Settling time weight & $7 / 8$ \\
\hline
\end{tabular}

Table 8. AODV specific parameters

\begin{tabular}{ll}
\hline Parameter & Value \\
\hline Lifetime of a route reply message & $10 \mathrm{~s}$ \\
Time for which a route is considered active & $10 \mathrm{~s}$ \\
Time before route request message is retired & $6 \mathrm{~s}$ \\
Time which the broadcast id for a forwarded & $6 \mathrm{~s}$ \\
route request is kept & \\
Number of route request retries & 3 \\
Maximum route request timeout & $10 \mathrm{~s}$ \\
Local repair wait time & $0.15 \mathrm{~s}$ \\
\hline
\end{tabular}

Table 9. LGF specific parameters

\begin{tabular}{ll}
\hline Parameter & Value \\
\hline Tuning factor & 1.5 \\
Max expiry & $15 \mathrm{~s}$ \\
Min expiry & $1.2 \mathrm{~s}$ \\
Topological scope & 3 \\
\hline
\end{tabular}

\subsection{Performance with varying pause time}

Figure 8 evaluates the reliability of packet delivery of the different routing protocols; LGF, GPSR, AODV and DSDV. In general, DSDV, GPSR and AODV perform better as the pause time used in the random way model increases. In contrast, LGF is more robust at higher mobility levels, although the results indicate that its packet delivery ratio is relatively poor when compare to other protocols at low mobility. This is largely due to the way in which LGF handles link failures. GPSR, AODV and DSDV optimise the handling of link failure for stale connectivity. In contrast in LGF, we drop packets as soon as we see the link fail. It was a design decision to decrease the average packet delay at the cost of reducing the delivery ratio, we describe this in more detail below.

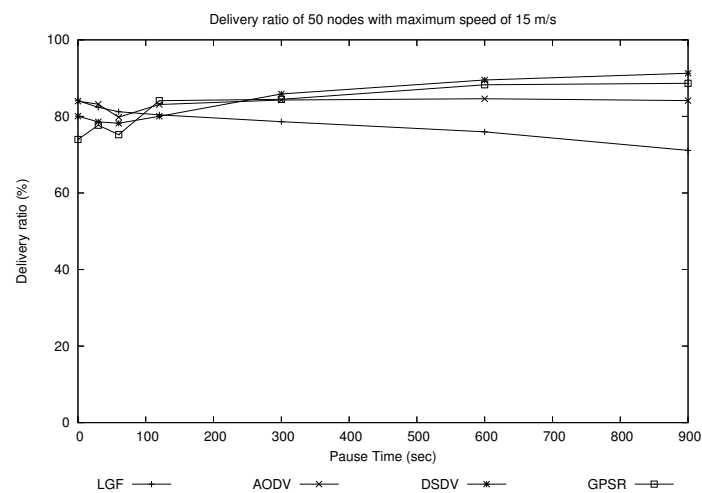

Figure 8. Comparison of the packet delivery ratio as a function of pause time

Both DSDV and AODV, upon notification of link retransmission failure, both protocols keep the packets in the buffer queue until the route becomes available again. This technique has not been published but it was found to be in the NS2 implementation. In the event of a link retransmission failure, GPSR applies the same technique used by DSR. It removes the routing entry of broken link before it en-queues the packet in the buffer for the routing protocol to forward the packet to a different next hop[1]. In LGF, the protocol drops the packet, updates the route entry, and propagates the broken link to other neighbouring nodes. Our results show that the link failure techniques used by GPSR, DSDV and AODV are opportunistic. The idea is to keep or redirect the packet when a link retransmission failure is encountered. Although this could increase the packet delivery ratio in some cases when connectivity is stable. However, in some scenarios such as where there is node mobility and the opportunity of direct or indirect re-delivery are not available, undelivered packets then linger for too long in the output buffer queue and can contribute to a higher average packet delay. Interestingly, our results show that the other protocols gain an advantage in the scenarios which use pause times of 300, 600 and 900 seconds. Current LGF design is unoptimised, we would expect to improve the performance of LGF in this respect.

This optimisation for increased packet delivery however does have side effects. From our observations, the average packet delay is increased as a results of this opportunistic delivery. In Figure 9, we show the effects on both AODV 


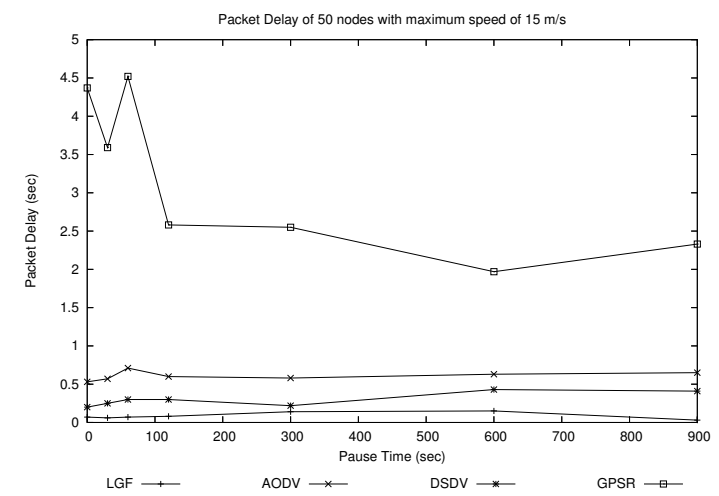

Figure 9. Comparison of average packet delay as a function of pause time

and DSDV are less significant as they only keep the undelivered packet for a short period of time. In contrast, GPSR retains the packet for much longer, this causes GPSR to have an increased delivery ratio, but this has the side effect of a higher average packet delay. Our results show LGF consistently has a lower latency than other routing protocols. LGF achieves this by not holding the packets in the event of link retransmission failure.

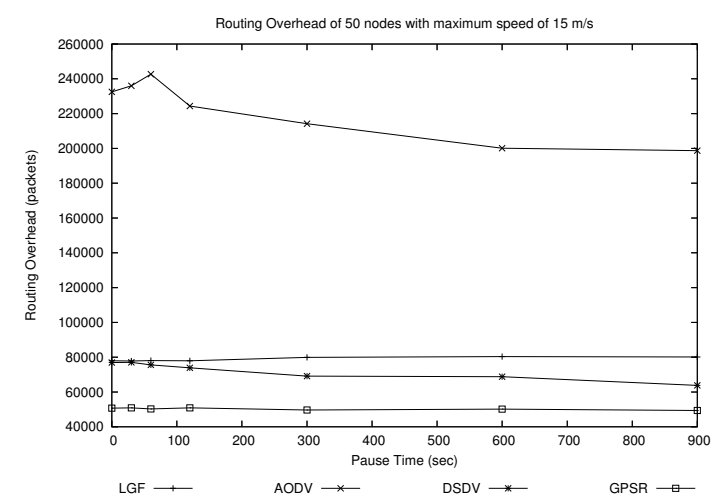

\section{Figure 10. Comparison of routing overhead as a function of pause time}

Figure 10 highlights the communications overhead of the different routing protocols. In LGF, the adaptive route update advertises more frequently in areas where link failure is more likely to occur while maintaining moderate updates in other areas where link failure is less likely to be encountered. Although, the advertisement is restricted to the local scope, LGF in general is sending out more frequent but restricted updates to its neighbours within its local scope. This explains why the overall communication overhead of LGF in this simulation is higher than DSDV. When com- pared with other protocols, LGF has lower communication overheads than reactive AODV but higher overheads than DSDV or GPSR. Despite its merit of having a low routing overhead, GPSR can encounter the effect of stale state when connectivity to its adjacent nodes changes more rapidly than its neighbours' periodic advertisements.

\subsection{Performance with varying velocity}

In this simulation, we tested performance of a system with 100 nodes over a wider area. Compared to previous simulations, the maximum distance between two nodes is larger, and therefore nodes are expected, on average, to take more hops between the source and destination. Additionally, the density of nodes in this simulation is 133 nodes per $\mathrm{km}^{2}$ as compared to the previous density of 111 nodes per $\mathrm{km}^{2}$. With more network overhead introduced as a result of the denser and larger system, it is further anticipated that contention and interference issues experienced in IEEE 802.11 networks could be more critical than previously measured. As a result, the channel capacity of the network is reduced[10] and consequently the average packet delay in general increases and the ratio of successful delivery decreases compared to previous simulations.

In comparison to other protocols, the results in figure 11 however do indicate that LGF is relatively steady and robust with respect to the measured delivery rate over a variety of velocities. We can conclude from these results that LGF is more reliable and adaptive to unsettled, dynamic topologies than other protocols.

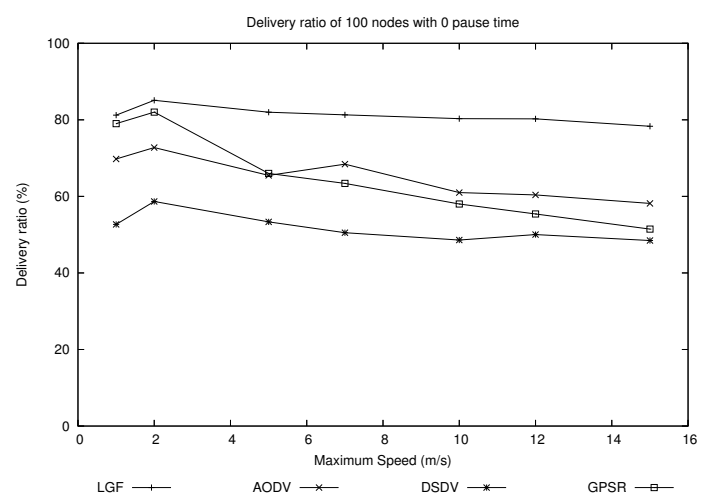

Figure 11. Comparison of packet delivery ratio as a function of maximum velocity

Our results in figure 12 shows that LGF performs consistently well with respect to routing overhead over a variety of velocities. These results are similar to the previous simulation results, the high communication overheads associated with reactive AODV is a result of a higher number of route 
discoveries and local repairs AODV is performing. Comparing with earlier results where we used less nodes and a smaller physical area, the overheads we observed are more onerous than in the previous simulation. Our observations show that the overheads associated with LGF are lower than the other protocols as the number of nodes is doubled from 50 to 100 . Because DSDV needs to maintain global state for all the nodes in the network, its overheads increase in proportion to the number of nodes in the network. In contrast, the restricted route update in LGF adapts well to the increased size of the network with the results confirming LGF's communication overheads scale better than the other protocols.

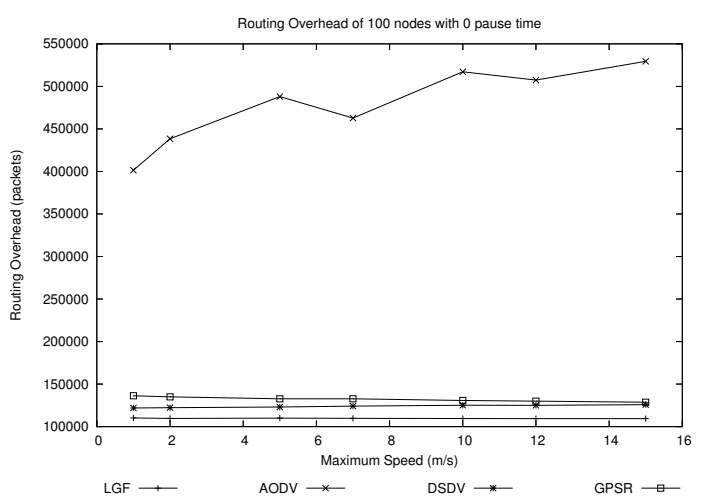

Figure 12. Comparison of the routing overheads as a function of maximum velocity

As shown in figure 13, DSDV does not converge fast enough to cope with the changes in connectivity for the scenario where it uses a periodic update timer of $15 \mathrm{sec}-$ onds, and when the network size has been increased. As a result of this, more undelivered packets are held in the queues in the network before they eventually expire and are dropped. Our results show the on demand path setup of AODV has a lower average packet delay than DSDV when simulating 100 nodes, this accounts for the performance advantage shown for the AODV local repair scheme in a dense network. If we consider the overall performance of all the protocol on packet delivery ratio, routing overheads and average packet delay, LGF provides a better overall balance performance than other protocols.

\subsection{Path length}

Figure 14 compares the path length for successful delivered packets for each protocol against the ideal shortest path retrieved from the NS2 simulator. The ideal shortest path is the shortest possible path only constrained by the physical radio range. The evaluation was carried out with a random way point mobility model using a 0 seconds pause

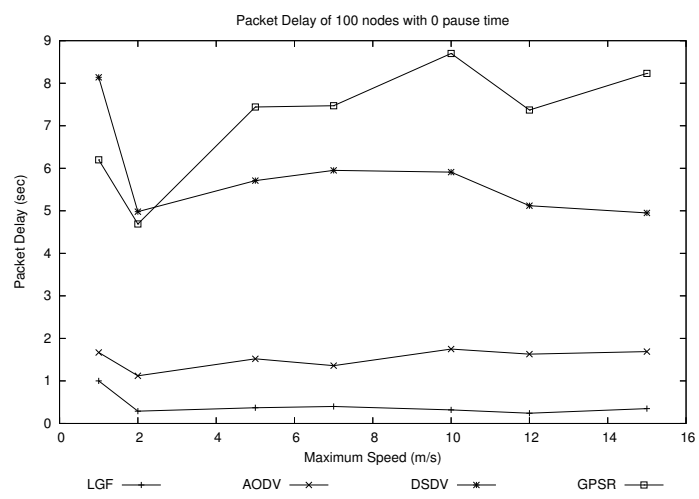

Figure 13. Comparison of average packet delay as a function of maximum velocity

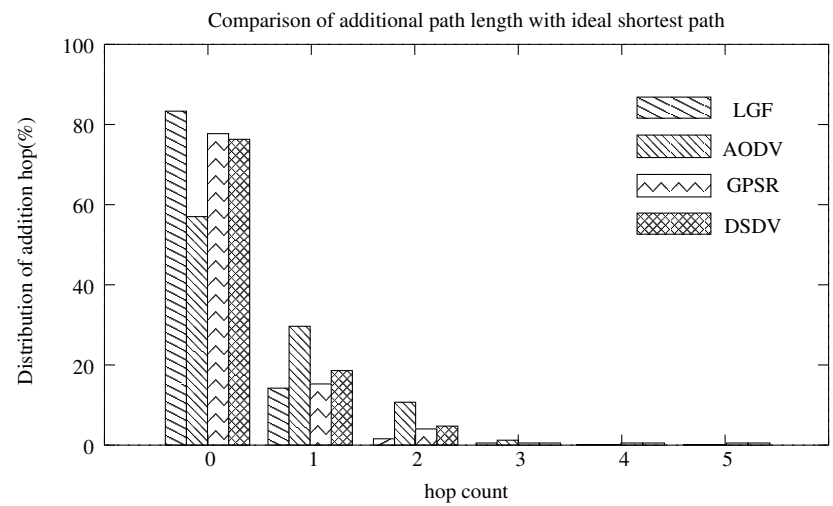

Figure 14. Comparison of the average path length for each of four protocols with ideal shortest path

time with a maximum velocity of $15 \mathrm{~m} / \mathrm{s}$ and 50 nodes placed randomly in area of $1500 \times 300 \mathrm{~m}^{2}$. The results indicate that LGF on average achieves $83.52 \%$ of optimal path length while GPSR obtains $78.98 \%$ of optimal path length. Although theoretically DSDV is supposed to maintain an optimal path, the slow update interval does not prevent misleading stale state from being used by the packet delivery mechanism. This results in sub-optimal routing. DSDV only routes $77.37 \%$ of its packets via the optimal path. Only $55.43 \%$ percent of AODV's packets are routed by the optimal path. A contributing factor to this is AODV's local repair algorithm which is fixing broken paths without considering what the alternative optimal path between the source and destination is.

\section{Conclusion}

In summary, we present a hybrid routing protocol, Landmark Guided Forwarding, which uses a restrictive hybrid 
advertisement at a rate regulated by its connectivity sensitive algorithm. LGF applies optimal routing when the destination is within its topological range, and systematically resolves a transient next hop through locally optimal resolution when an optimal route is unavailable.

We ran simulations with 50 nodes and 100 nodes, the results indicate the overheads of LGF scale better than other protocols when the number of nodes is double from 50 to 100. In our performance evaluation of varying pause time, it is apparent that route optimisations by AODV, DSDV and GPSR do improve the packet delivery ratio when rate of change of the topology is low, when using a mobility model where the pause time is greater than 120 and has a maximum velocity of $15 \mathrm{~m} / \mathrm{s}$. However the simulation results allow us to conclude that these optimisations could have the side effect of a higher average packet delay. The effect is more pronounced when simulated with 100 nodes, and a slightly wider network diameter. In contrast, LGF is able to maintain a steady, swift and reliable delivery even in the presence of a higher probability of unstable network connectivity. When comparing the path length with other protocols, LGF, surprisingly, has the highest score of the protocols under consideration.

In conclusion, local optimal routing sidesteps the constraint of maintaining a globally optimal path, as generally required by existing MANET protocols. This results in LGF being a relatively scalable and robust protocol with low overheads as compared to other MANET routing protocols, and yet, somewhat surprisingly, it retains relatively short routes nonetheless.

\section{Future work}

In future work, we wish to look into formal verification and setting up a testbed for LGF. In addition, we would like to investigate the use of a coordinate system with the Landmark Guided Forwarding protocol, to exploit the common goals of reducing routing overheads. Internet coordinate systems such as Lighthouses [15] and Virtual Landmarks [17] could be used to supplement the process of selecting the topologically closest node to the destination. LGF could additionally exploit the topological data from the coordinate systems to avoid routing errors when removing edges or nodes that violate the triangle inequality.

\section{References}

[1] B.Karp and H.T.Kung. GPSR: Greedy perimeter stateless routing for wireless networks. In Proceedings of 6th Annual Internation Conference on Mobile computing and Networking(MobiCom 2000), Boston, MA, USA, pages 243-254, Feb 2000.
[2] L. Blazevic, S. Giordano, and J.-Y. L. Boudec. Self organized terminode routing. Cluster Computing, 5(2):205-218, 2002.

[3] P. Bose, P. Morin, I. Stojmenovic, and J. Urrutia. Routing with guaranteed delivery in ad hoc wireless networks. Wireless Networks, 7(6):609-616, 2001.

[4] C.Perkins and P.Bhagwat. Highly dynamic destinationsequenced distance-vector routing(DSDV) for mobile computing. Computer Communication Review, 24(4):234-244, Oct 1994.

[5] D.Johnson, D.A.Maltz, and J.Broch. DSR: The Dynamic Source Routing Protocol For Multihop Wireless Ad Hoc Networks, Ad Hoc Networking. Addison-Wesley Longman Publishing Co. Inc., Boston, MA, 2001.

[6] N. group at ISI. Ns 2 home page. World Wide Web, http: //www.isi.edu/nsnam/ns/.

[7] Z. J. Haas and M. R. Pearlman. ZRP: a hybrid framework for routing in Ad Hoc networks. Book chapter in Ad Hoc Networks, Editor C. Perkins. Addison-Wesley Longman Publishing Co., Inc., 2001.

[8] Y. Kim, J.-J. Lee, and A. Helmy. Modeling and analyzing the impact of location inconsistencies on geographic routing in wireless networks. SIGMOBILE Mob. Comput. Commun. Rev., 8(1):48-60, 2004.

[9] A. LaMarca, Y. Chawathe, S. Conolvo, J. Hightower, I. Smith, J. Scott, T. Sohn, J. Howard, J. Hughes, F. Potter, J. Tabert, P. Powledge, G. Borriello, and B. Schilt. Place lab: Device positioning using radio bacons in the wild. Technical report, Intel Research, 2004.

[10] J. Li, C. Blake, D. S. J. D. Couto, H. I. Lee, and R. Morris. Capacity of ad hoc wireless networks. Mobile Computing and Networking, pages 61-69, 2001.

[11] J. Li, J. Jannotti, D. De Couto, D. Karger, and R. Morris. A scalable location service for geographic ad hoc routing. In Proceedings of the 6th ACM International Conference on Mobile Computing and Networking (MobiCom 'O0), pages 120-130, Aug. 2000.

[12] M. H. Lim, A. Greenhalgh, J. Chesterfield, and J. Crowcroft. Hybrid routing: A pragmatic approach to mitigating position uncertainty in geo-routing. Technical report, University of Cambridge, 2005.

[13] E. M.Royer and C. K. Toh. A review of current routing protocols for ad hoc mobile wireless networks. IEEE Personal Communications Magazine, pages 46-55, April 1999.

[14] C. Perkins and E. M. Royer. Ad hoc on demand distance vector routing. In Proceedings of the 2nd IEEE Workshop on Mobile Computing Systems and Applications, New Orleans, LA., pages 90-100, February 1997.

[15] M. Pias, J. Crowcroft, S. Wilbur, S. Bhatti, and T. Harris. Lighthouses for scalable distributed location. In Second International Workshop on Peer-to-Peer Systems (IPTPS '03), Feb 2003.

[16] C. E. S.Dass and E. M.Royer. Performance comparison of two on-demand routing protocols for ad hoc networks. IEEE Personal Communication Magazine, special issue on mobile Ad Hoc networks, 8(1):16-29, Feb 2001.

[17] L. Tang and M. Crovella. Virtual landmarks for the internet. In Proceedings of Internet Measurement Conference 143152, Miami Beach, FL., October 2003. 